\title{
Quality management systems in radiology
}

\author{
G K Korir, ${ }^{1}$ BSc, MSc, PhD; J S Wambani, ${ }^{2} \mathrm{MB}$ ChB, MMed (Rad), Pediatric (Rad); I K Korir, ${ }^{3}$ BSc, MSc, PhD; \\ M Tries, ${ }^{1}$ BS (Physics), MS, PhD; B Mulama, ${ }^{2}$ MB ChB, MMed (Diagnostic Rad)
}

${ }^{1}$ Department of Physics and Applied Physics, University of Massachusetts Lowell, Lowell, Massachusetts, USA

${ }^{2}$ Radiology Department, Kenyatta National Hospital, Nairobi, Kenya

${ }^{3}$ National Nuclear Regulator, Centurion, South Africa

Corresponding author: G K Korir (ggkk382@yahoo.com)

Background. The use of X-ray imaging is ever increasing in proportion to the need for radiological services and the latest technology. Quality management of patient radiation dose monitoring is fundamental to safety and quality improvement of radiological services.

Objective. To assess the level of quality management systems in medical X-ray facilities in Kenya.

Methods. Quality management inspection, quality control performance tests and patient radiation exposure were assessed in 54 representative X-ray medical facilities. Additionally, a survey of X-ray examination frequency was conducted in 140 hospitals across the country.

Results. The overall findings placed the country's X-ray imaging quality management systems at $61 \pm 3 \%$ out of a possible $100 \%$. The most and the least quality assurance performance indicators were general radiography X-ray equipment quality control tests at $88 \pm 4 \%$, and the interventional cardiology adult examinations below diagnostic reference level at $25 \pm 1 \%$, respectively.

Conclusions. The study used a systematic evidence-based approach for assessing national quality management systems in radiological practice in clinical application, technical conduct of the procedure, image quality criteria, and patient characteristics as part of the quality management programme.

S Afr J Rad 2013;17(3):84-88. DOI:10.7196/SAJR.886

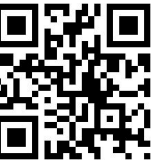

Rapid advances in medical imaging evince a consequent need for timely and high-quality anatomical information of the subject's body. High disease prevalence and the population explosion are additional compounding factors that increase the workload upon an insufficient number of skilled medical personnel, especially in developing countries. ${ }^{[1,2]}$ The performance and financial circumstances of radiology departments in the healthcare systems of Level four (HCL IV) countries $^{[3]}$ may be compromising the quality of patient care because of a low appreciation of quality management in operational efficiency, image quality and patient radiation dose. There is therefore a need to benchmark the level of quality management systems to provide evidence-based records aimed at improving safety and ensuring quality healthcare. In Kenya, few studies on quality management in radiology have been reported, ${ }^{[4]}$ despite the need for in-depth studies to develop effective, efficient and sustainable programmes commensurate with the socio-economic status of the country.

The broad legal requirements for quality assurance (QA) and quality control (QC) of medical imaging equipment and ancillary equipment exist in most developed countries. ${ }^{[5-9]}$ Developing countries have yet to fully establish adequate guidelines to match those in developed countries, especially for X-ray equipment performance and image quality criteria, inter alia. ${ }^{[10,11]}$ In addition, a shortage of data from developing countries means that information is absent about radiology QA procedures, the organisational framework for the performance of QC tests, evaluation and institution of corrective measures. The present study was therefore initiated to benchmark the national quality management system (QMS) and compare the results with established international safety standards from the International Atomic Energy Agency (IAEA), the International
Commission on Radiological Protection (ICRP), the Health Protection Agency (HPA) in the UK, the Commission of European Communities (CEC), and the American College of Radiology (ACR). ${ }^{[12-16]}$

\section{Methods \\ Quality management in radiology \\ Radiology practice}

This study did not involve patients directly; it was approved by the Kenyatta National Hospital Ethics and Research Committee. A total of 269 (90\% coverage) radiology departments and private clinics in the country were sent letters via the administrator in charge of each institution, requesting them to participate and also to complete a QA questionnaire. The level of quality management per radiological facility was determined by scoring the quality indicators grouped into the following 3 main categories: human resource control, physical asset control, and safety of the work environment. The presence or absence of the quality factors mentioned in the questionnaire response provided by the facilities, or during the author's inspection, led to the award of a score of 1 (pass) or 0 (fail), respectively.

\section{QA administration}

The overall performance evaluated as QA (16 points) constituted the summation of scores from:

- Category one (Human Resource Control). Seven points: patient records, workers' licences, patient shielding, professional association, patient dosimetry, QA manual and in-service training.

- Category two (Physical Asset Control). Four points: QC programme, equipment service reports, QC results, and equipment licence.

- Category three (Safety of Work Environment). Five points: 
public safety, workers' safety, personnel monitoring, radiation warning signs and appointed radiation safety officer.

The facility QA administration rating was calculated as a percentage: the number of scores passed divided by the total 16 metrics considered. Patient dose was calculated the same way, from the total of scores of one point if the measured dose were below the available diagnostic reference levels (DRLs), or zero if it were above the DRLs.

\section{National quality management} level and index

The 15 QA indicators listed in Table 1 were as follows: QA administration performance, X-ray QC test performance (general radiography (GR), mammography, computed tomography (CT)), image quality (IQ) performance (mammography, GR), and children and adult patient dose below DRLs (GR, mammography adults only, general fluoroscopy (GF), interventional radiology (IR) adults only, interventional cardiology (IC) adults only, CT). Each quality indicator was scored and an average score calculated. The maximum score was $100 \%$ for each indicator, bringing the total maximum sum of percentage scores to 1500 . The national QA level was calculated as an average of the percentage scores from the $15 \mathrm{QA}$ indicators. Performance ratings were awarded as follow: an average score $\geq 75 \%$ was excellent, 50 $74 \%$ was good, 26 - $49 \%$ was fair, and $\leq 25 \%$ was poor.

The national quality management index (QMI), which has a maximum score of 1 , was calculated by dividing the percentage score from each QA indicator by the sum of all percentage points scored in the QA indicators. For the QMI, a score $\geq 0.082$ was rated as excellent, $0.054-0.081$ as good, 0.027 -0.053 as fair, and $\leq 0.026$ as poor.

\section{Results \\ Radiology practice comparison with rest of world}

The survey response was provided by 140 representative X-ray facilities, equivalent to $47 \%$ of the total number of operating facilities in Kenya. A total of 54 (20\%) representative X-ray facilities were visited to make QA presentations, QC measurements, in-person observations of radiological examinations, to interact with hospital staff, and to encourage participation in the survey. This was a large-scale study with the overall sample size of both patients and radiation facilities meeting the $95 \%$ confidence level and $5 \%$ confidence interval.

The frequency of the number of X-ray procedures in the year 2011 was $82 / 1000$ of the general Kenyan population. These statistics, when combined with the data in Table 2, indicate that each radiologist was responsible for approximately 325000 examinations/year in Kenya. When general medical practitioners were included, each medical practitioner was responsible for approximately $8100 \mathrm{X}$-ray examinations/year. The radiographer-patient workload was 189300 examinations/year. The average annual case workload/X-ray machine was as follows: GR 4000 ; mammography 700; GF 2 000; interventional procedures (IPs) 300; and CT 3500 patients. The number of qualified medical physicists responsible for monitoring equipment performance, image quality and estimating patient dose was largely inadequate.

\section{QA administration}

\section{Human resource control}

The $38 \%$ overall quality performance in this category based on operator qualification and practice was fair. All workers at the facilities considered were recognised by their respective professional body; $32 \%$ were authorised by the National Regulatory
Table 1. Quality management performance of Kenyan radiological facilities ${ }^{[4,10,18,19,20]}$

\begin{tabular}{lll}
\hline Quality indicators & Score (\%) & QMI \\
\hline QA administration performance & 37 & 0.040 \\
GR quality control tests performance & 88 & 0.096 \\
Mammography quality control tests & 82 & 0.089 \\
GR image quality performance & 60 & 0.066 \\
Mammography image quality performance & 81 & 0.088 \\
CT image quality performance & 61 & 0.067 \\
GR adults examinations below DRLs & 86 & 0.093 \\
Mammography examinations below DRLs & 77 & 0.083 \\
GF adult examinations below DRLs & 70 & 0.076 \\
CT adults examinations below DRLs & 40 & 0.044 \\
GR paediatric examinations below DRLs & 81 & 0.089 \\
GF paediatric examinations below DRLs & 60 & 0.065 \\
Interventional radiology adult examinations below DRLs & 38 & 0.041 \\
Interventional cardiology adult examinations below DRLs & 25 & 0.027 \\
CT paediatric examinations below DRLs & 33 & 0.036 \\
National QM performance level & $\mathbf{6 1}$ & $\mathbf{0 . 0 6 7}$ \\
Total & 918 & 1 \\
CT = computed tomography; DRL = diagnostic reference levels; GF = general fluoroscopy; GR $=$ general &
\end{tabular}

Table 2. Level of provision of medical radiology staff and facilities per million people $\mathrm{e}^{[3,17]}$

\begin{tabular}{|c|c|c|c|c|c|c|c|c|}
\hline Personnel/facilities & $\begin{array}{l}\text { Kenya* } \\
(2011)\end{array}$ & $\begin{array}{l}\text { Ghana } \\
(2010)\end{array}$ & $\begin{array}{l}\text { Uganda } \\
(2010)\end{array}$ & $\begin{array}{l}\text { UNSCEAR } \\
\text { HCL IV }\end{array}$ & $\begin{array}{l}\text { Britain } \\
(1983)\end{array}$ & $\begin{array}{l}\text { France } \\
(1982)\end{array}$ & $\begin{array}{l}\text { Netherlands } \\
\text { (1983) }\end{array}$ & UNSCEAR HCL I \\
\hline Medical doctors & 120 & 140 & 86 & 45 & 1400 & 2090 & 1400 & 2800 \\
\hline Radiologists & 3 & 1 & 1 & 0.1 & 28 & 91 & 84 & 110 \\
\hline Medical physicists & 0.6 & 1 & 0.2 & - & - & - & - & - \\
\hline Radiographers & 5 & 8 & 7 & - & 143 & 340 & 330 & - \\
\hline $\mathrm{X}$-ray equipment & 20 & 10 & 4 & 4 & 198 & 244 & 310 & 290 \\
\hline Mammography & 0.5 & 0.3 & 0.2 & 0.1 & - & - & - & 24 \\
\hline
\end{tabular}


Authority to administer ionising radiation for medical purposes. No facility possessed a written QA manual that took into consideration radiation safety issues concerning patients, workers and the public. There were inadequate thyroid, eye and gonad shields in the facilities that used high-radiation modalities such as CT and the IPs. None of the facilities used protective shielding on body regions not scanned during CT scan examination. Similar results were noted for the need to document patient preparation procedures in the institutional QA manual. All the radiological facilities kept patient records that included name, weight, age, type of procedure and date of examination. However, there was only one facility that recorded CT and mammography doses; the rest did not, despite the fact that X-ray machines, like CT scanners, display patient dose per examination.

\section{Physical asset control}

The $24 \%$ overall performance in this category was poor. Only $22 \%$ of the facilities had reports of semi-annual preventive maintenance consisting of only the QC tests. Only $68 \%$ of the X-ray facilities had received an equipment operating licence in 2011. The availability of QC programs scored lowest among the facilities that participated in the study, followed by the availability of QC records.

\section{Safety of work environment}

The 57\% overall performance in this category was good. A total of $72 \%$ of the facilities had radiation warning signs posted in conspicuous places. The requirements of monitoring radiation workers monthly for radiation dose and facility safety inspections were adhered to by $61 \%$ of the facilities. Only $22 \%$ of the facilities had an appointed radiation safety officer, of whom $30 \%$ reported having been sponsored for refresher courses. Individual facility performance figures indicated that $39 \%$ were good, $28 \%$ were fair, and $33 \%$ were poor, resulting in the overall national rating of 38\% in the radiology QA administration section.

\section{Patient dose assessment}

Patient dose assessment was estimated according to the details obtained from over $10000 \mathrm{X}$-ray examinations collected from over 54 facilities. ${ }^{[10,18-20]}$ The patient composition was: 4514 (45\%) children and 5522 (55\%) adults. The patient dose data were distributed according to imaging modalities as follows: GR 4803 patients (48\%); mammography 1252 patients (12\%); GF 493 patients (5\%); IPs 310 patients (3\%); and CT scan 3178 patients (32\%).

\section{National QM level}

Table 1 provides the overall QA performance for medical radiological facilities in Kenya. The overall level of QA was determined to be $60 \%$. For the various categories, the GR X-ray efficiency performance achieved the level of excellence at $84 \%$, adult patient dose (68\%) and image quality (61\%) were good, and the QA administration (37\%) and patient dose in adults were fair. None of the general quality indicators was rated as poor.

\section{Discussion}

\section{National quality management level}

The competent authority in Kenya responsible for radiation safety is the Radiation Protection Board, which was established in 1986 through the Radiation Protection Act, Cap 243 of the Laws of Kenya. ${ }^{[21]}$ The Radiation Protection Board via legislation authorises the use of radioactive materials and radiation-producing devices at established or new facilities, establishes dose limits, and may conduct inspections and apply enforcement. Regulations have been set up that cover notification and authorisation, dose limits, and control of medical exposure. The current Radiation Protection Act, Cap 243, is being revised as the Radiation Safety Bill; it addresses additional regulatory requirements, but the acquisition, safety culture and proper use of modern radiological equipment is still dependent on the expertise of diagnostic imaging professionals within the facility. The situation poses a challenge to hospitals on how to establish a comprehensive QA programme for medical exposure with the participation of a limited number of qualified experts in radiation physics, as well as taking quality management principles, established locally and/or internationally, into account.

Effective use of medical equipment at the hospital level may be limited by cost, technical expertise and the level of co-ordination among personnel. The Medical Practitioners and Dentist Board (MPDB) has a well-established and critical role in regulating the health sector in Kenya. However, there has been no reported co-ordination and development of radiological guidelines or any quality management studies conducted for diagnostic or therapeutic procedures in the country. Moreover, the protocols for developing standards and guidelines in radiology are yet to be established. The Radiation Protection Act generally requires irradiating devices in use in the country to comply with International Safety Standards. ${ }^{[13,14]}$ To compensate for the regulatory deficiencies, diagnostic imaging professionals within the country have indicated that standards for X-ray equipment performance tests, as well as referral guidelines, need to be established and mandated in a hospital QA manual, in terms of regulations and/or by licence conditions.

The regulatory authority practice of outsourcing radiation protection services to board-certified service providers without a national guideline on X-ray equipment performance does not adequately address the country's radiology quality management needs. The board certifications for dosimetry service providers must involve the diagnostic imaging community to establish guidelines that are being initiated through this study. The dosimetry service provision guidelines will not be limited to the standards for calibration and verification of radiation measuring instruments, but will also include monitoring, evaluations and corrective measures for the dosimetry service provider and the hospital diagnostic radiology departments. The radiation safety assessment within a QA manual should include a comprehensive account of X-ray equipment, staff qualifications, occupational radiation exposure monitoring, health status and integrity of structural shielding for the actual use of personal protective devices for staff and comforters, and the use of radiation protection devices for patients, among others. All these radiation protection parameters require regular checks, status confirmation, and the keeping of records. Total QA can be promoted through dynamic departmental organisation structure, standard operating procedures, and the application of locally or internationally recognised standards. ${ }^{[2,23]}$

These practices will facilitate organisation, integration and the assigning of responsibilities to specific departmental professionals. The level of QA/QC roles and responsibilities will therefore be inextricably linked to a professional contribution to quality improvement of clinical practice through evidence-based audits and reviews, as well as followup of corrective measures. The radiologists or medical practitioners in charge should oversee the establishment of local quality systems guided by local practice as well as international standards that are currently available in most developed countries. The process of establishing local 
quality management systems may offer an opportunity to local radiology departments and hospitals to collaborate with established radiological institutions in developed countries. In addition to comprehensive QA systems, there should be effective QA committees, professional certification, quantitative measurements, equipment standards, imaging guidelines, technical capacity building, and continuous professional development. ${ }^{[24]}$ Incident and accident investigation procedures as well as reporting procedures should also be established at both the hospital and the national level.

The diagnostic imaging professionals indicated being affiliated with the MPDB, the Kenya Association of Radiologists, the East African Association for Radiation Protection, and the Society of Radiographers in Kenya. The majority of radiological facilities involved in this study did not appreciate the critical role of patient dosimetry as contributing to a QA programme. The use of protective gear to cover body areas outside the region of interest while performing CT procedures was low. There was a general assumption that the protective gear was meant for radiation workers and those who assist unco-operative or incapacitated patients. This view resulted in low adherence to the use of basic protective gear, including the absence of a breast garment of thinly layered bismuth impregnated with radio-protective latex for covering the irradiated region not imaged. ${ }^{[25,26]}$

A critical evaluation of the data provided by the hospitals revealed divergent perspectives on the contents and details of a written QA manual in radiology. There was a need for a standard format in preparing a QA manual, and documenting patient preparation, the nature of radiological examinations, patient details, and potential risks and benefits, all in a consistent database format. According to NCRP Report 99, proficient performance by imaging personnel can be enhanced by monitoring activities, and the establishment of policies and controlled record keeping systems that provide objective evidence of personnel performance. ${ }^{[27]}$ Record keeping of equipment performance and maintenance was poor. Most facilities kept equipment assessment technical reports after maintenance and repair work on the radiological devices, but these were brief and appropriate only for billing purposes. The engineers' service and maintenance reports are similar in format and content to medical physicists' equipment QC test reports that include proper equipment identification, documentation of performance tests results, and comments. There is a need to train imaging professionals and engineers in the initiation of regular performance verification, including the use of consistency and reproducibility checks to ensure that systematic errors are promptly detected and corrected. The resultant low performance level was attributed to the lack of medical physicists in the country and hence the absence of QA/QC programmes and compliance audits. Radiology departments should institute an effective QA programme and train QC technologists. Large hospitals, such as the teaching and referral or specialised centres, should consider the establishment of a QA committee whose primary function would be to maintain effective quality management systems among all groups associated with medical X-ray imaging.

The low number of workers' X-ray equipment operating licences reported in most facilities was associated with delayed inspections, delayed application to the issuing authority, and slow processing of licences by the regulator, or stringent requirements for licensing. The performance level in the occupational radiation protection measures and the posting of radiation warning signs was found to be in good standing. Radiation safety officers were encouraged to scrutinise all relevant QA reports including monthly personnel radiation dose measurements, so that the best practices are identified and adopted for routine use.

The superior performance in X-ray equipment quality control over QA administration in this study supports a central theme of medical imaging based on accomplishing diagnosis within a reasonable time, with minimum equipment variables, and improved patient dose management. In contrast, the QA administration components constitute the quality elements that are dependent on cost, awareness and co-ordination of health administrators, imaging professionals, regulatory authorities and X-ray equipment vendors. Imaging professionals should therefore assert their roles and responsibilities in QA because they are inextricably linked to the quality of their product and service in the supply chain. Whereas the shared mission of quality patient care is noted, the results from this study also indicate that the low interdependence of QA and IQ can be improved if radiologists oversee the establishment of comprehensive QM systems, including effective QM committees, professional certification, quantitative measurements, equipment standards, imaging guidelines, technical capacity, and continuous professional development guided by the application of recognised international standards. The patient dose being generally above the published DRLs from developed countries emphasises that the DRLs, which are a quality assurance tool, should be specific to a particular medical imaging task for a group of patients in a specified region or local state of practice.

In this study, the inclusion of radiation exposure to patients emphasises the importance of the national optimisation process in $\mathrm{X}$-ray imaging practice as well as ensuring staff safety. In setting an optimisation strategy for a country, it is important that the choice of examinations be based on collective dose contribution and the frequency of the examinations, to maximise the overall benefit to the population. Analysis of patient dose, and review of DRLs, should be performed at least twice a year. Local diagnostic reference levels (LDRLs) are dynamic and always changing with respect to current hospital equipment and the examinations performed within a specific facility.

The QMI is derived from the scores accumulated from quality indicators that quantify performance in a step-by-step evaluation process. QMI is relevant to employees, managers, patients, equipment manufacturers, health authorities and regulatory authorities. QMI can enhance problem identification and teamwork within an institution.

\section{Conclusion}

The present study has set out the status/level of the scientific and technical knowledge available towards integrating quality management and instituting effective radiological protection of patients in clinical practice. The study shows how to merge the role of hospital administrators, use of equipment performance tests and patient dose measurements without compromising clinical images, while achieving radiological protection. The information presented provides for a systematic evidence-based approach for the development of radiological practice guidelines that specify clinical purpose, technical conduct of the procedure, image quality criteria, unique patient characteristics, and other appropriate factors including the radiological protection of patients as part of a QA programme.

We determined in this study that QA compliance in Kenyan medical imaging facilities is good, but with room for improvement. At the end of the study period, each participating facility received an evaluation report and recommendations. Seminar presentations were 


\section{ORIGINAL ARTICLE}

prepared for imaging professionals, equipment suppliers, and hospital administrators. The study thus provides baseline information that supports the urgent need for comprehensive QA via training, adequate regulations, professional certifications, clinical image quality guidelines, and accreditation of medical imaging facilities. The QMI is a unique performance assessment tool, suitable for HCL IV countries such as Kenya, for regularly measuring, monitoring and benchmarking the national quality improvement in radiology.

Acknowledgements. We sincerely thank the management and radiology staff of the hospitals that participated in the IAEA Project (RAF/9/033: Strengthening Radiological Protection of Patient and Medical Exposure Control), and the IAEA for their support.

1. Wambani JS, Korir GK, Onditi EG, Korir IK. A Survey of computed tomography imaging techniques and patient dose in Kenya. East Afr Med J 2010;87(10):400-407.

2. Korir GK, Wambani JS, Korir IK. Establishing quality assurance baseline for radiological protection of patients in diagnostic radiology. S Afr J Rad 2011;15(3):70-79.

3. United Nations Scientific Committee on the Effects of Atomic Radiation. Sources and Effects of Ionizing Radiation; UNSCEAR 2008 Report Vol. 1; Annex A: Medical Radiation Exposures. New York: United Nations Publications, 2010.

4. Korir GK,Wambani JS, Korir IK. Establishing quality management baseline in the use of computed tomography machines in Kenya. J Appl Clin Med Phys 2012;13(1):187-196. [http://dx.doi.org/10.1120/ jacmp.v13i1.3457]

5. Environment Protection Authority. Registration Requirements \& Industry Best Practice for Ionizing Radiation Apparatus used in Diagnostic Imaging, Radiation Guidelines; Test protocols for parts 2 - 5 . Sydney: New South Wales Environment Protection Authority, 2000

6. International Atomic Energy Agency. Dosimetry in Diagnostic Radiology: An International Quality Assurance Manual, Technical Report Series No. 457. Vienna: IAEA, 2007.

7. National Council on Radiation Protection and Measurements. Quality Assurance for Diagnostic Imaging, NCRP Report No.99. Bethesda: NCRP, 1988.

8. American Association of Medical Physicists. Quality Control of Diagnostic Radiology, AAMP Report No. 74. New York: AAPM, 2002.

9. Institute of Physical Sciences in Medicine (IPSM). Dosimetry Working Party of the Institute of Physical Sciences in Medicine. National Protocol for Patient Dose Measurements in Diagnostic Radiology. Chilton: NRPB, 1992
10. Korir GK, Wambani JS, Korir IK. Patient doses using multidetector computed tomography scanners in Kenya. Radiat Prot Dosimetry 2012;151(2):267-271. [http://dx.doi.org/10.1093/rpd/ncr484]

11. Muhogora WE, Ahmed NA, Almosabihi A, et al. Patient doses in radiographic examinations in 12 countries in Asia, Africa, and Eastern Europe: Initial results from IAEA projects. Am J Roentgenol 2008:190(6):1453-1461.

12. International Atomic Energy Agency. International Basic Safety Standards for Protection against Ionizing Radiation and for the Safety of Radiation Sources, Safety Series No. 115. Vienna: IAEA, 1996.

13. International Commission on Radiological Protection. The 2007 Recommendations of the International Commission on Radiological Protection. Annals of the ICRP Publication 103. Oxford: Pergamon Press, 2007.

14. International Commission on Radiological Protection. Recommendations of the International Commission on Radiological Protection. Annals of the ICRP Publication 60. Oxford: Pergamon Press, 1991.

15. European Commission. European Guidelines on Quality Criteria for Diagnostic Radiographic Images, European Commission Report 16260. Luxembourg: European Commission, 1996.

16. American College of Radiology. Technical Standard for Medical Physics Performance Monitoring of Radiographic and Fluoroscopic Equipment. Reston: American College of Radiology, 2001:729-732.

17. Contento G, Malisan MR, Padovani R, Maccia C, Wall BF, Shrimpton PC. A comparison of diagnostic radiology practice and patient exposure in Britain, France and Italy. Br J Radiol 1988;61(722):143-152.

18. Wambani JS, Korir GK, Bwonya SMN, Korir IK. Assessment of patient doses during mammography practice at Kenyatta National Hospital. East Afr Med J 2011;88:368-378.

19. Wambani JS, Korir GK, Korir IK, Kilaha S. Establishment of local diagnostic reference levels in paediatric screen-film radiography at a children's hospital. Radiat Prot Dosimetry 2013;154:465-476.

20. Korir GK. Quality Assurance and Radiation Protection for Medical X-Ray Procedures in Kenya. PhD dissertation. Lowell: University of Massachusetts Lowell, 2013.

21. The Radiation Protection Act, Chapter 243 Laws of Kenya. The Radiation Protection (Standards) Regulations, Legal Notice No. 54. Nairobi: Government Printers, 1986.

22. American Association of Physicists in Medicine. Managing the use of Fluoroscopy in Medical Institutions, AAPM Report No. 58. New York: AAPM, 1998.

23. International Electrotechnical Commission. Evaluation and routine testing in medical imaging departments: Part 2 - 6 Constancy Tests X-Ray Equipment for Computed Tomography IEC 1223-2-6. Geneva: International Electrotechnical Commission, 1994.

24. Korir GK, Wambani JS, Korir IK. A Pilot study on Quality Assurance Performance in Computed Tomography Facilities in Kenya, Proceedings of the XIV Convención de Ingeniería Eléctrica, Presentación SI-10. Las Villas: 2011; CD ISBN 978-959-250-685-5.

25. Beaconsfield T, Nicholson R, Tornton A, Al-Kutoubi A. Would thyroid and breast shielding be beneficial in CT of the head. Eur Radiol 1998;8:664-667.

26. Hopper KD, King SH, Lobell ME, Tenttore TR, Weaver JS. The breast: In-plane x-ray protection during diagnostic thoracic CT-shielding with bismuth radioprotective garments. Radiology 1997;205:853-858.

27. National Council on Radiation Protection and Measurements. Quality Assurance for Diagnostic Imaging, NCRP Report No.99. Bethesda: NCRP, 1988. 